\section{Berechnung der Korrekturfaktoren}

Nach Bestimmung der zeitabhängigen Zuckerwerte in der Hydrolysat-Kurve $Z(t)$ und der Abbaurate $\alpha$ der entsprechenden reinen Komponente unter den gewählten Standardbedingungen, kann mit Hilfe eines der oben besprochenen Verfahren die Zuckergesamtmenge $F_{\infty} \approx F_{T} \approx Y(0)$ in der biologischen Probe berechnet werden. $F_{\infty} / Z(\tau)$ ergibt den Korrekturfaktor für quantitative Untersuchungen unter ähnlichen Bedingungen; $Z(\tau)$ ist dabei der zur fest- gelegten Zeit $\tau$ bestimmte Zuckerwert im Hydrolysat (s. Tab. 2 und Abb. 3). Die mit der beschriebenen Methode ermittelten Hydrolyse-Korrekturfaktoren einiger in den untersuchten Proben vorhandenen Zucker sind in Tab. 2 angegeben.

TH. GH. möchte der Alexander von Humboldt-Stiftung, Bad Godesberg, B.R. Deutschland, seinen Dank für das Verleihen eines Dozentenstipendiums aussprechen. Die Autoren danken der Deutschen Forschungsgemeinschaft für die gewährte Unterstützung.

\title{
Proteine als Schutzstoffe gegenüber dem Gefriertod der Zelle
}

\section{U. Heber und M. Kempfle}

\author{
Botanisches Institut der Universität Düsseldorf und \\ Institut für Physiologische Chemie der Universität Bonn \\ (Z. Naturforsch. 25 b, 834-842 [1970] ; eingegangen am 3. April 1970)
}

\begin{abstract}
Two protein factors from hardy leaves were highly active in preventing the inactivation of photophosphorylation of washed thylakoid membranes which takes place during freezing of thylakoids in the absence of protective compounds. Non-hardy leaves did not yield protective protein material. On a unit weight basis, protection by the protein factors was 10 to 100 times better than protection by compounds of low molecular weight such as sucrose, glycerol or dimethylsulfoxide. Very low amounts of the protein factors, which alone were scarcely protective, considerably reduced the concentration of sucrose required for the complete protection of thylakoids during freezing. The protein factors were heat-stable and had a molecular weight between 10000 and 20000 daltons. It is assumed that they contribute to and are in part responsible for the frost tolerance of hardy plant material.
\end{abstract}

Bekanntlich sind eine Reihe von Pflanzen vor allem aus gemäßigten und kalten Klimagebieten in der Lage, Resistenz gegenüber tiefen Temperaturen $\mathrm{zu}$ entwickeln. Während der Wachstumsphase sind sie in der Regel frostempfindlich, in der Dormanzperiode - im Winter - sind sie resistent. Biochemische Veränderungen in der intakten Zelle während des Resistenzerwerbs sind lange Gegenstand von Untersuchungen gewesen, ohne daß es gelang, klare und eindeutige kausale Beziehungen aufzudecken, die es gestatten würden, die Ursache der Resistenz befriedigend zu erklären ${ }^{1}$. Erst die Untersuchung des Einflusses tiefer Temperaturen auf Zellfraktionen führte zu folgenden Aussagen: Gefrieren verändert die Permeabilität von ungeschützten plasmatischen Membranen ${ }^{2}$. In Mitochondrienmembra-

1 J. Leviti: The Hardiness of Plants, Academic Press, New York 1956.

2 U. Heber, Plant Physiol. 43, 1343 [1967].

3 U. Heber u. K. A. Santarius, Plant Physiol. 39, 712 [1964].

4 P. Mazur, Annu. Rev. Plant Physiol. 20, 419 [1969]. nen führen gefrierbedingte Permeabilitäts-Änderungen zum Zusammenbruch der oxydativen Phosphorylierung, in Chloroplastenmembranen zur Inaktivierung der Photophosphorylierung ${ }^{2,3}$. In anderen Plasmamembranen sind ebenfalls drastische Veränderungen biologischer Eigenschaften zu erwarten ${ }^{4}$. Insgesamt genügen die beobachteten Veränderungen an Membranen, den Frosttod der Zelle zu erklären. Schutzstoffe vermögen die gefrierbedingten Permeabilitäts-Veränderungen zu verhindern ${ }^{3}$. Schutzstoffwirksamkeit besitzen unter anderem niedermolekulare Zucker, Zuckeralkohole und Glycerin, aber auch unphysiologische Verbindungen wie Dimethylsulfoxyd $^{15}$. Damit erklärt sich die Beobachtung einer Anhäufung von Zuckern in vielen, wenngleich nicht allen frostresistenten Pflanzengeweben ${ }^{1,6}$ und von

5 U. Heber u. R. ERnst, in: Cellular Injury and Resistance in Freezing Organisms, E. Asahina ed., Bd. II, S. 63, Hokkaido University, Sapporo 1967.

6 A. SAKaI, Contrib. Inst. Low Temperat. Sci. Hokkaido Univ. B 11, 1 [1962]. 
Glycerin in einigen überwinternden Insekten $^{7,8}$. Bisher ist nichts darüber bekannt, ob auch hochmolekulare Zellbausteine als Schutzstoffe in Frage kommen.

Die nachfolgende Arbeit beschreibt die Isolierung und einige Eigenschaften zweier Protein- bzw. Polypeptid-Fraktionen aus frostharten Pflanzen, die eine vielfach höhere Schutzstoffwirksamkeit im Membrantest besitzen als die bekannten niedermolekularen Schutzstoffe. Über einen Teil der Ergebnisse wurde kurz in Vorträgen berichtet ${ }^{5,9,10}$.

\section{Experimentelles}

\section{Material}

Die meisten Schutzstoffisolierungen wurden an Blättern von frosthartem Spinat (Spinacia oleracea L., Sorte Matador oder Feldmaterial unbekannter Herkunft) durchgeführt. Isolierungen erfolgten daneben auch aus Blättern von Winterweizen (Triticum sativum Lam., Feldmaterial), Winterroggen (Secale cereale L., Feldmaterial) und Rapünzchen (Valerianella olitoria Mönch.) und aus Rinde von Pappeln (Populus nigra L.). Das Material wurde zwischen Ende Dezember und Anfang März, also in frosthartem Zustand, geerntet. Sofern es nicht sofort zur Aufarbeitung kam, wurde es bei $-30{ }^{\circ} \mathrm{C}$ aufbewahrt.

\section{Isolierung}

Blatt- oder Rindenmaterial wurde in $150 \mathrm{~g}$ Portionen in je $250 \mathrm{ml}$ eines Puffers folgender Zusammensetzung für 2 bis $3 \mathrm{~min}$ in einem Starmix-Gerät homogenisiert: $0,05 \mathrm{M}$ Tris(hydroxymethyl)-aminomethan, $10^{-2} \mathrm{M} \mathrm{KH}_{2} \mathrm{PO}_{4}, 0,3 \mathrm{M} \mathrm{NaCl}, 10^{-2} \mathrm{M}$ Ascorbat und $3 \cdot 10^{-3} \mathrm{M}$ Cystein, $\mathrm{pH}$ 8. Grobe Partikel wurden durch Filtrieren durch 8 Lagen Verbandsmull, Membranfragmente durch 20 bis 30 min Zentrifugieren bei $50000 \mathrm{~g}$ entfernt. Der bräunlich gefärbte Überstand wurde vorsichtig mit $\mathrm{HCl}$ auf $\mathrm{pH} 4$ gebracht und das entstehende Sediment abzentrifugiert. Es verblieb eine gelblich gefärbte Lösung, die in der Regel, aber nicht immer, zur Präzipitierung von Proteinmaterial geringer Schutzstoffaktivität für 2 min auf $95^{\circ} \mathrm{C}$ erhitzt wurde. Der Schutzstoff erwies sich als stabil gegenüber Erwärmen. Nach dem Abkühlen wurde $67 \mathrm{~g}\left(\mathrm{NH}_{4}\right)_{2} \mathrm{SO}_{4}$ $\mathrm{zu}$ je $100 \mathrm{ml}$ Lösung zugesetzt und die resultierende Fällung abzentrifugiert. Der Überstand wurde verworfen, die Fällung in wenig Wasser aufgenommen. Nach Einstellen des $\mathrm{pH}$ auf 7,8 wurde bei $0{ }^{\circ} \mathrm{C}$ gegen $0,005 \mathrm{M}$ tris (Hydroxymethyl)-aminomethan $(\mathrm{pH} \quad 7,8)$

7 E. Asahina, in: Cryobiology, H. T. Meryman ed., S. 451, Academic Press, London 1966.

8 R. W. SAlt, in: Handbook of Physiology, Section 4, Amer. Physiol. Soc., Wash., D.C. 1964. ein bis zwei Tage dialysiert, ungelöstes bzw. ausgefallenes Material durch Zentrifugieren entfernt und die verbleibende Lösung gefriergetrocknet. Zur Dialyse wurde Cellophanschlauch von Kalle \& Co., WiesbadenBiebrich, verwendet.

Zur weiteren Fraktionierung wurde das gefriergetrocknete Material in wenig Wasser gelöst $(100 \mathrm{mg} / \mathrm{ml})$ und auf eine mit Sephadex-G-25 gefüllte Säule (Höhe $35 \mathrm{~cm}$, Durchmesser $2,6 \mathrm{~cm}$ ) gegeben, die vorher mit 0,005 м Tris (hydroxylmethyl) aminomethan $(\mathrm{pH} \quad 7,8)$ equilibriert worden war. Zur Entwicklung diente $0,005 \mathrm{~m}$ Tris (hydroxymethyl) aminomethan, $\mathrm{pH} \quad 7,8$. Das Eluat wurde in 2,5-ml-Anteilen aufgefangen, deren Absorption bei $280 \mathrm{~nm}$ gemessen wurde. Ein typisches Elutionsprofil ist in Abb. 1 wiedergegeben. Die Hauptfraktion $\left(\mathrm{F}_{1} \mathrm{G}_{25}\right)$ war schutzstoffaktiv, ebenso die nachfolgende 2. Fraktion $\left(\mathrm{F}_{2} \mathrm{G}_{25}\right)$. Allerdings war diese klein und wurde deshalb nicht näher untersucht. Die dritte Fraktion $\left(\mathrm{F}_{3} \mathrm{G}_{25}\right)$, die nicht in allen Trennungen scharf ausgeprägt erschien, besaß kaum noch Schutzstoffaktivität gegenüber gewaschenen Thylakoiden. Zwei relativ große, am Ende erscheinende Fraktionen $\left(\mathrm{F}_{4} \mathrm{G}_{25}\right.$ und $\left.\mathrm{F}_{5} \mathrm{G}_{25}\right)$ waren leicht gelblich gefärbt. Sie waren inaktiv im Schutzstofftest und wurden nicht näher charakterisiert. Ihr Absorptionsspektrum weist auf einen Gehalt an Flavonoiden hin. Die Hauptfraktion wurde gefriergetrocknet und anschließend erneut auf einer mit Sephadex G-50 beschickten Säule fraktioniert. In der Regel ergab sich eine scharfe Trennung in zwei Unterfraktionen (Abb. 2). Beide waren schutzstoffaktiv, wobei die Aktivität der nachfolgenden Fraktion $\left(F_{2} G_{50}\right)$ höher war als die der vorangehenden $\left(F_{1} G_{50}\right)$. Die Fraktion $F_{1} G_{50}$ wurde nach Gefriertrocknung erneut auf einer mit Sephadex-G-75 gefüllten Säule aufgetrennt. Eine scharfe Trennung wurde hier gelegentlich nicht erreicht, doch ließ sich immer eine vorlaufende Fraktion $\left(F_{1} G_{75}\right)$ von einer nachlaufenden $\left(F_{2} G_{75}\right)$ separieren. Ein Profil für eine Sephadex-G-75-Trennung ist in Abb. 3 dargestellt. Die Fraktionen $\mathrm{F}_{2} \mathrm{G}_{50}, \mathrm{~F}_{2} \mathrm{G}_{75}$ und $F_{1} G_{75}$ wurden gefroren, gefriergetrocknet, in wenig Wasser aufgenommen und zur Entfernung von Puffer gegen Wasser bei $0{ }^{\circ} \mathrm{C}$ dialysiert. Anschließend wurden sie wieder eingefroren und gefriergetrocknet.

Zur Gewinnung von Schutzstoff aus Plastiden wurden Chloroplasten im gleichen Medium wie oben angegeben durch $15 \mathrm{sec}$ Homogenisieren aus Blättern freigelegt. Das Homogenisat wurde filtriert und $5 \mathrm{~min}$ bei $200 \mathrm{~g}$ sedimentiert. Der resultierende Überstand wurde $12 \mathrm{~min}$ bei $600 \mathrm{~g}$ zentrifugiert. Das Sediment bestand aus Chloroplasten, die unmittelbar oder nach einer Waschung im gleichen Puffer durch Aufnehmen in Wasser osmotisch geschockt wurden. Dabei wurde der Schutzstoff aus dem Thylakoidsystem ausgewaschen. Die weitere Aufarbeitung erfolgte nach Entfernen der Membrananteile wie oben für Blätter angegeben.

9 U. Heber, Cryobiology 5, 188 [1968].

10 U. Heber, in: Ciba Fdn. Symp. „The Frozen Cell“, p. 175, J. \& A. Churchill, London 1969. 


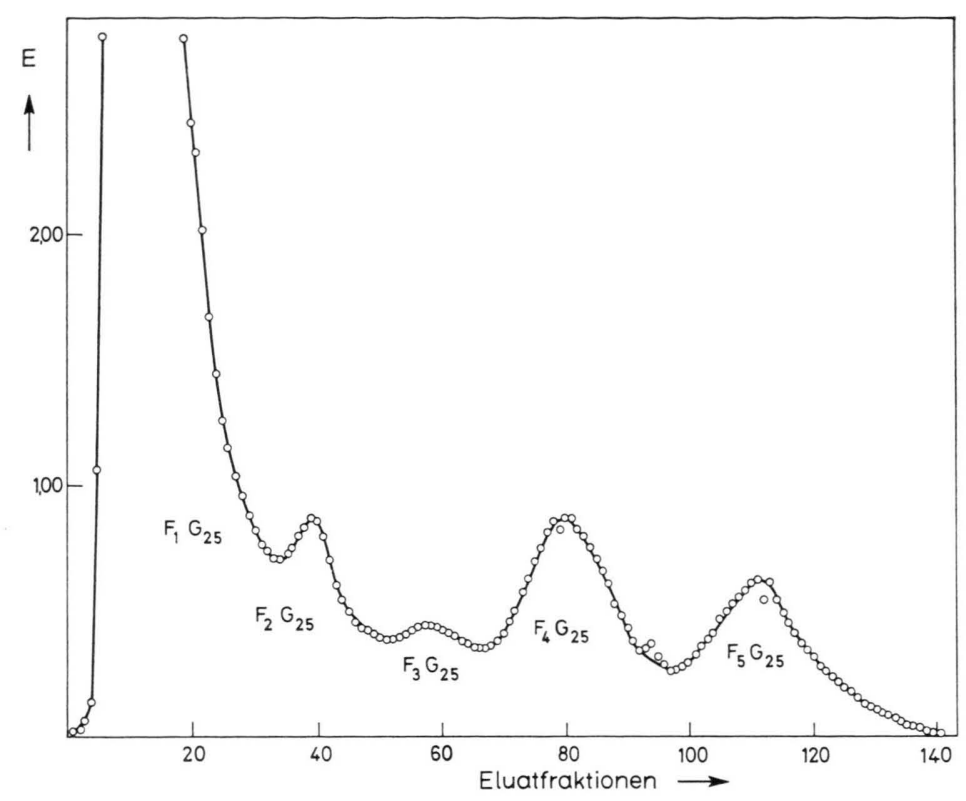

Abb. 1. Elutionsprofil einer hitzestabilen Protein- oder Polypeptidfraktion aus Blättern von frosthartem Spinat nach Passage durch Sephadex G-25. Zeichenerklärung s. Text. Ordinate: Extinktion der Eluate bei $280 \mathrm{~nm}$. Abszisse: Reihenfolge der Eluatfraktionen.

Als Testsystem zur Bestimmung der Schutzstoffaktivität dienten 2-mal mit Wasser in der Ultrazentrifuge gewaschene Chloroplastenmembranen (Thylakoide). Die Photophosphorylierung solcher Membranen wird durch 3-stdg. Gefrieren bei $-25{ }^{\circ} \mathrm{C}$ stark herabgedrückt, in Gegenwart von ca. $0,03 \mathrm{M} \mathrm{NaCl}$ oder mehr

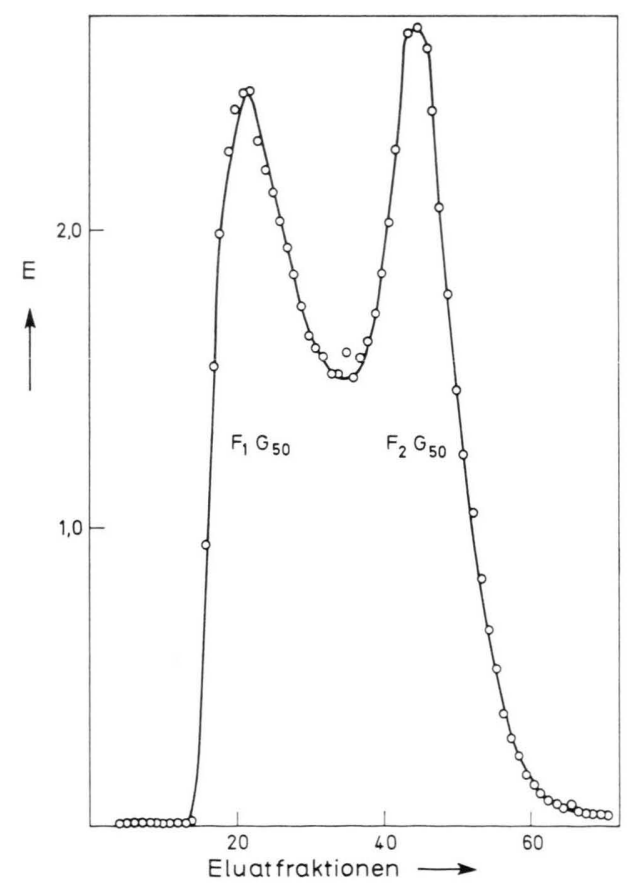

Abb. 2. Elutionsprofil der Fraktion $F_{1} G_{25}$ (s. Abb. 1) nach Passage durch Sephadex G-50. sogar vollkommen inaktiviert. Wird vor dem Gefrieren Schutzstoff zugesetzt und dann nach einer Präinkubationsperiode von 20 min gefroren, so bleibt die Fähigkeit zur ATP-Synthese - abhängig von der Menge des zugesetzten Schutzstoffes - mehr oder weniger vollständig erhalten. Die Zusammensetzung des Testsystems und die Gewinnung der Thylakoide wurde an anderer Stelle beschrieben ${ }^{2,3}$. Die Konzentration an $\mathrm{NaCl}$ in der Thylakoidsuspension vor dem Gefrieren war in der Regel ca. $5 \cdot 10^{-3} \mathrm{M}$.

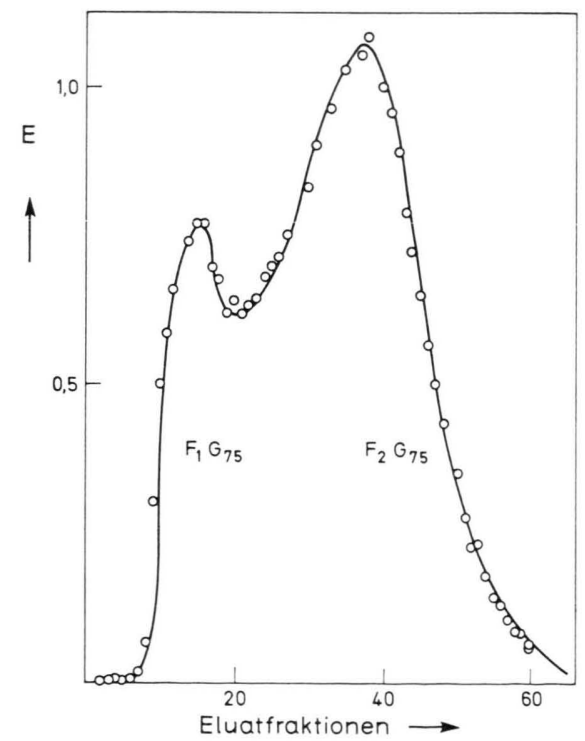

Abb. 3. Elutionsprofil der Fraktion $F_{1} G_{50}$ (s. Abb. 2) nach Passage durch Sephadex G-75. 
Ergebnisse

\section{Schutzstoffaktivität}

Verschiedene niedermolekulare Verbindungen können biologische Membranen gegen den Einfluß des Gefrierens schützen. Zu den wirksamsten Stoffen zählen Glycerin, Dimethylsulfoxyd und Saccharose. Tatsächlich werden die beiden erstgenannten Verbindungen auch zur Gefrierpräservierung von Erythrozyten und anderen Zellen verwendet. Abb. 4

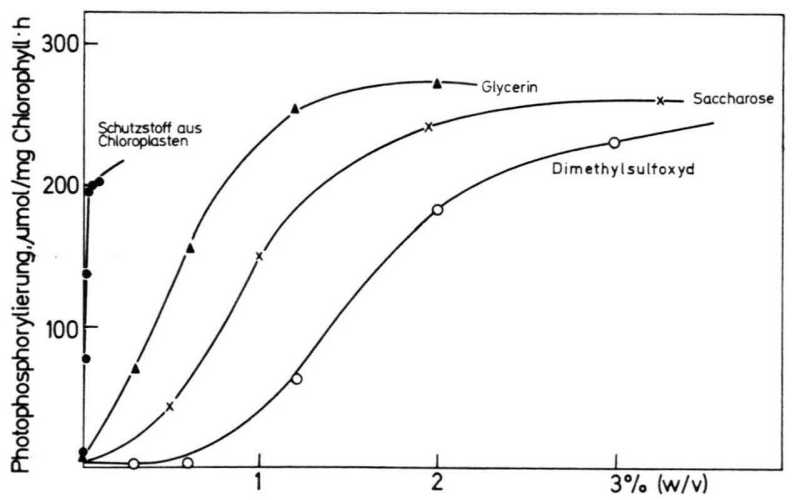

Abb. 4. Schutzwirkung von hochmolekularem Schutzstoff aus Chloroplasten gegenüber der Gefrierinaktivierung von Thylakoidmembranen im Vergleich zur Wirkung von Saccharose, Glycerin und Dimethylsulfoxyd. Thylakoide wurden in Gegenwart verschiedener Mengen Schutzstoff für $3 \mathrm{Stdn}$. bei $-25{ }^{\circ} \mathrm{C}$ gefroren. Anschließend wurde die Photophosphorylierungs-Kapazität der Membranen gemessen. Photophosphorylierung bei $0^{\circ} \mathrm{C}$ gehaltener Kontrollen: $270 \mu \mathrm{mol} / \mathrm{mg}$ Chlorophyll $\cdot \mathrm{h}$.

zeigt die Wirksamkeit von hochmolekularem Schutzstoff, der durch Ammonsulfat-Fraktionierung aus isolierten Chloroplasten von frosthartem Spinat gewonnen wurde, im Gefriertest mit Thylakoidmembranen im Vergleich zu den genannten niedermolekularen Verbindungen. Es ist ersichtlich, daß der hochmolekulare Faktor den anderen Stoffen weit, bei Bezug auf das Trockengewicht bis zu einem Faktor von 100 , überlegen ist. Allerdings wird beobachtet, daß kompletter Schutz (Erreichen der Phosphorylierungsrate, die in den ungefrorenen Kontrollen gefunden wird) durch den hochmolekularen Faktor auch im Sättigungsbereich oft nicht erreicht wird. Das ist wahrscheinlich dadurch bedingt, daß das zum Test verwandte Thylakoidsystem für den hochmolekularen Faktor trotz der Präinkubation aus sterischen Gründen nicht vollständig „zugänglich“ ist, wohl aber für Saccharose, die bei höheren Konzentrationen komplett zu schützen vermag. Tatsächlich wurde wiederholt gefunden, daß eine Präinkubation der Thylakoide mit dem Faktor dessen Wirksamkeit erhöhte ${ }^{4}$.

Die Wirksamkeit des aus isolierten Chloroplasten gewonnenen Schutzstoffes wurde in der Regel von den Fraktionen aus Blättern nicht ganz erreicht, wenngleich diese noch um ein Vielfaches aktiver waren als Saccharose oder andere niedermolekulare Schutzstoffe. Abb. 5 zeigt die Wirksamkeit der Frak-

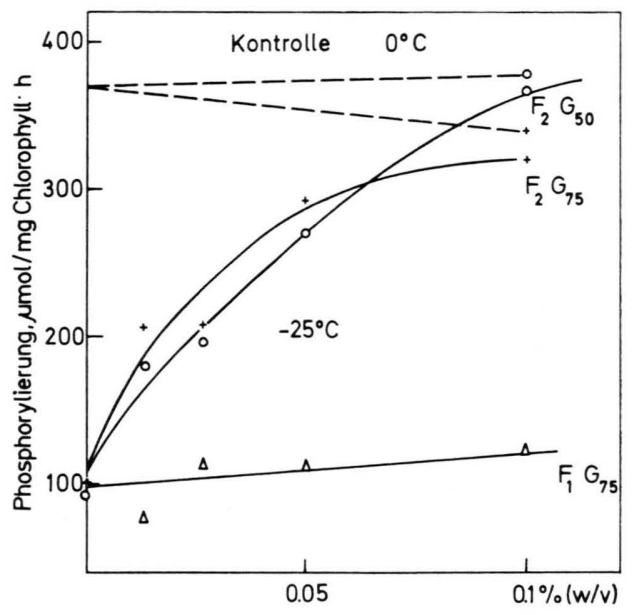

Abb. 5. Schutzwirkung verschiedener Proteinfraktionen im Membrantest. Bedingungen wie in Legende zu Abb. 4.

tionen $\mathrm{F}_{2} \mathrm{G}_{50}, \mathrm{~F}_{2} \mathrm{G}_{75}$ und $\mathrm{F}_{1} \mathrm{G}_{75}$ im Vergleich. Während $F_{2} G_{50}$ und $F_{2} G_{75}$ sich in ihrer Aktivität nicht wesentlich unterschieden $-\mathrm{F}_{2} \mathrm{G}_{50}$ war oft etwas wirksamer - besaß die Fraktion $\mathrm{F}_{1} \mathrm{G}_{75}$ nur geringe Schutzstoffaktivität. Andere Proteinfraktionen aus Blättern erwiesen sich im untersuchten Konzentrationsbereich als unwirksam. Schutzstoffaktive Fraktionen $F_{2} G_{50}$ und $F_{2} G_{75}$ wurden nicht nur aus Spinatblättern, sondern in vergleichbarer Aktivität auch aus Blättern von Winterweizen, Winterroggen und Rapünzchen und auch Pappelrinde isoliert. In den beiden letzteren Fällen erwies sich die Gegenwart von dunkel gefärbten Oxydationsprodukten als störend. Auch Hemmeffekte auf die Photophosphorylierung von Thylakoidmembranen wurden beobachtet.

Die Inaktivierung der Phosphorylierung von Thylakoidmembranen durch Gefrieren ist zeitabhängig ${ }^{3}$. Es bestand die Möglichkeit, die überraschend hohe Schutzstoffwirkung der aktiven Fraktionen lediglich über eine Verlangsamung des Inaktivierungsprozesses zu erklären. Diese Möglichkeit trifft nicht zu, wie sich aus Abb. 6 ergibt. Auch über längere Gefrierzeiten hin blieb der Schutzeffekt konstant. 


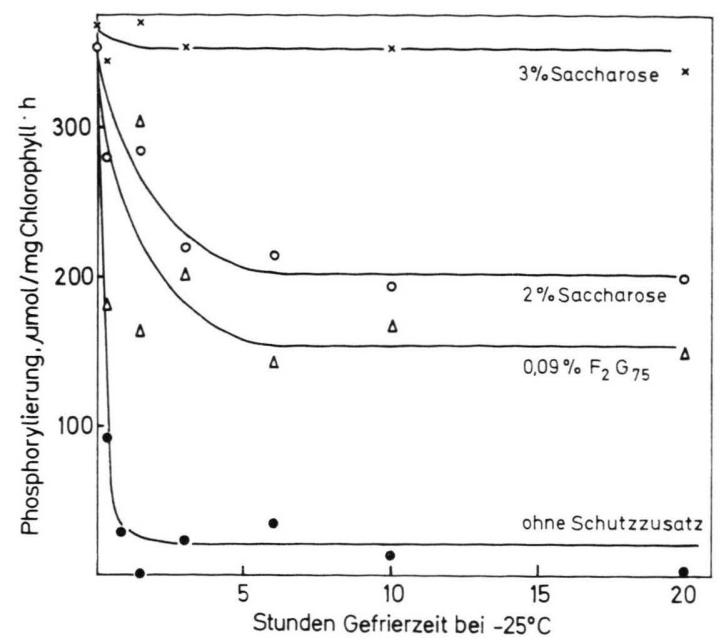

Abb. 6. Abhängigkeit der Schutzwirkung von Saccharose und $\mathrm{F}_{2} \mathrm{G}_{75}$ von der Gefrierdauer bei $-25^{\circ} \mathrm{C}$.

Die aktiven Fraktionen vermögen ebenso wie Zucker Thylakoide nicht nur gegenüber Gefrieren bei $-25^{\circ} \mathrm{C}$ (Standardtest) zu schützen, sondern auch gegenüber schnellem oder langsamem Frieren auf die Temperatur flüssigen Stickstoffs.

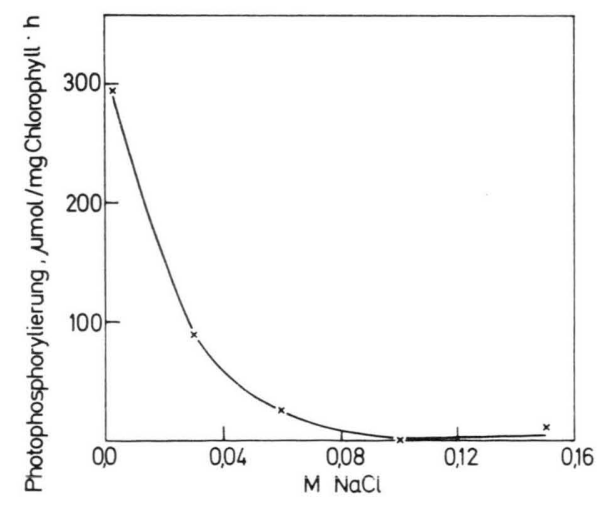

Abb. 7. Aufhebung der Schutzwirkung von $0,15 \% \mathrm{~F}_{2} \mathrm{G}_{75}$ auf Thylakoidmembranen durch Zusatz von $\mathrm{NaCl}$ vor dem $\mathrm{Ge}$ frieren. Gefrierdauer: $3 \mathrm{Stdn}$. bei $-25^{\circ} \mathrm{C}$.

Salze beeinträchtigen die Schutzstoffwirkung. In Abb. 7 ist gezeigt, daß Zusatz steigender Mengen an $\mathrm{NaCl}$ vor dem Gefrieren die Schutzstoffwirkung der aktiven Fraktionen fortschreitend vermindert bzw. bei erhöhter Salzkonzentration aufhebt. Ganz ähnliche Beobachtungen wurden auch bei der Untersuchung der Schutzwirkung von Saccharose ${ }^{3}$, Glycerin oder Dimethylsulfoxyd gemacht.

Von Interesse ist das Verhalten der Schutzstoffe in Gegenwart von Zuckern. In Abb. 8 ist der Einfluß sehr niedriger Konzentrationen an $\mathrm{F}_{2} \mathrm{G}_{75}$ von Spinat auf die Schutzwirkung von Saccharose dargestellt. Konzentrationen an $\mathrm{F}_{2} \mathrm{G}_{75}$, die selbst nur eine sehr geringe Schutzwirkung auszuüben vermögen $(0,01 \%)$, bewirken eine deutliche Herabsetzung des zum Schutze des Systems erforderlichen Saccharosespiegels. Bereits geringfügig erhöhte Konzentrationen sind entsprechend effektiver. Der hochmolekulare Schutzstoff und Saccharose wirken also nicht unabhängig voneinander. Diese Verhältnisse sind offenbar von Bedeutung für die in vivo erforderlichen Schutzstoffkonzentrationen.

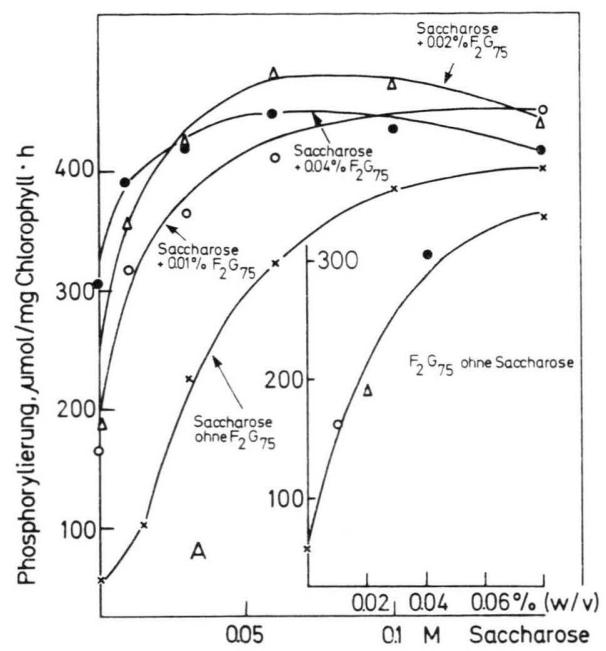

Abb. 8. A. Schutz von Thylakoidmembranen gegen die Einwirkung des Frierens durch Saccharose in Abwesenheit und in Gegenwart geringer Mengen an $\mathrm{F}_{2} \mathrm{G}_{75}$. B. Schutz von Thylakoidmembranen durch $\mathrm{F}_{2} \mathrm{G}_{75}$ allein. Gefrierzeit: $3 \mathrm{Stdn}$. bei $-25^{\circ} \mathrm{C}$.

\section{Spezifität der Schutzstoffwirkung}

In einigen Isolierungen (Spinat, Roggen) war die Aktivität der Fraktionen erheblich niedriger als in der Regel beobachtet wurde. Weiter hatten Fraktionen aus im Sommer geernteten, frostempfindlichen Spinat keine Schutzstoffwirksamkeit. Dies kann als erster Hinweis darauf gewertet werden, daß die Fraktionen nicht einheitlich sind. Das Fehlen von Schutzstoffaktivität im untersuchten Sommermaterial mag andeuten, daß die Schutzstoffe nur im Winter gebildet werden. Innerhalb der Fraktionen liegen also wahrscheinlich schutzwirksame und schutzunwirksame Anteile vor. Das spricht zunächst für eine hohe Spezifität der Schutzstoffwirkung. Jedoch bedeutet diese offenbar nicht, daß die Schutzstoffwirkung im Sinne eines „Schlüssel-Schloß“-Prinzips zu verstehen sei. Gegen eine solche Annahme spricht 
der Befund, daß selbst Pepton eine Schutzstoffwirkung ausübt, die erheblich gesteigert werden kann, wenn man Pepton der gleichen Fraktionierungsprozedur unterwirft, die für die Isolierung der Schutzstoffe aus Blättern beschrieben wurde. Bei der Sephadex-Trennung einer vorher mit Ammoniumsulfat fraktionierten Probe käuflichen Peptons (Pepton von Merck) ließ sich zwar keine Auftrennung in definierte Fraktionen erreichen, doch ergab ein entsprechend der Fraktionierung der Blattextrakte vorgenommenes „Schneiden“ der Eluate eine Peptonfraktion $\mathrm{F}_{2} \mathrm{G}_{50}$, deren Aktivität in Abb. 9 darge-

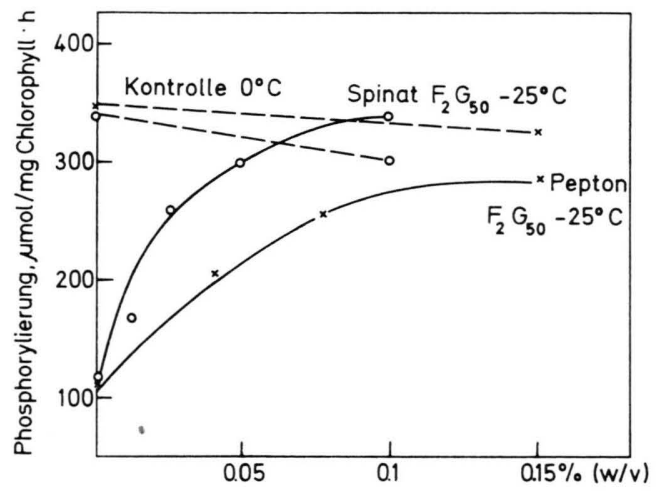

Abb. 9. Schutzwirkung von $F_{2} G_{50}$ und einer entsprechend isolierten Fraktion aus Pepton von Merck im Membrantest. Bedingungen wie in Legende zu Abb. 4.

stellt ist. Obgleich die Wirksamkeit der Blattfraktionen nicht erreicht wird, so sind offenbar auch unspezifisch gebaute Polypeptide zu hoher Schutzwirkung befähigt, die die niedermolekularer Schutzstoffe wie etwa Glycerin oder Saccharose erheblich übersteigt.

\section{Eigenschaften der Fraktionen}

Abb. 10 zeigt Spektren der aktiven Fraktionen. Ein Maximum der Absorption wurde bei etwa 275 $\mathrm{nm}$ beobachtet. Eine Nucleinsäurekomponente, die früher in schutzwirksamen Fraktionen enthalten war ${ }^{5}$, trat nach Sephadex-Fraktionierung nicht mehr in Erscheinung und ist am Schutzeffekt nicht beteiligt. Ein kleines Nebenmaximum bei etwa $340 \mathrm{~nm}$, das gelegentlich, vor allem bei Schutzstoffisolierungen aus Chloroplasten, auch fehlte, rührt wahrscheinlich von Verunreinigungen durch Flavonoide her.

Wie bereits für den Isolierungsgang angegeben, können die Schutzstoffe durch Ammoniumsulfat ausgesalzen werden. Die Schutzstoffaktivität wurde

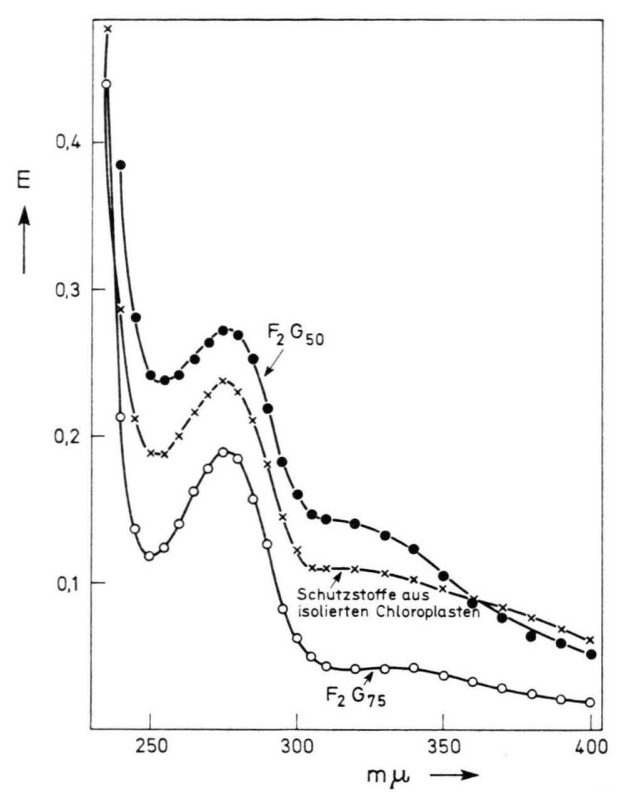

Abb. 10. UV-Spektren der Schutzstofffraktionen aus frostharten Spinatblättern.

durch kurzfristiges Erhitzen auf $100{ }^{\circ} \mathrm{C}$ nicht merklich beeinflußt. Zugabe von Trichloressigsäure zu den Fraktionen $F_{2} G_{50}, F_{2} G_{75}$ und $F_{1} G_{75}$ bewirkte eine starke Fällung, die im Überschuß des Fällungsmittels löslich war. Sulfosalicylsäure verursachte lediglich eine Trübung. Werte aus Stickstoffbestimmungen an den Fraktionen sind in Tab. 1 wieder-

\begin{tabular}{lc}
\hline Material & $\begin{array}{c}{[\%],, \text { Protein“" }} \\
(=6,25 \cdot \% \mathrm{~N})\end{array}$ \\
\hline $\mathrm{F}_{2} \mathrm{G}_{50}$ aus Spinat & 78 \\
$\mathrm{~F}_{2} \mathrm{G}_{50}$ aus Winterroggen & 80 \\
$\mathrm{~F}_{2} \mathrm{G}_{75}$ aus Spinat & 99 \\
$\mathrm{~F}_{2} \mathrm{G}_{75}$ aus Winterroggen & 97 \\
$\mathrm{~F}_{1} \mathrm{G}_{75}$ aus Spinat & 63 \\
$\mathrm{~F}_{1} \mathrm{G}_{75}$ aus Winterroggen & 64 \\
${ }_{\text {,F }} \mathrm{F}_{50}$ " abgetrennt aus Pepton Merck & 86 \\
\hline
\end{tabular}

Tab. 1. Der Gehalt der Fraktionen $F_{2} G_{50}, F_{2} G_{75}$ und $F_{1} G_{75}$ an „Protein“. Stickstoffbestimmung nach $\mathrm{Kj}$ el $\mathrm{dahl}$.

gegeben. Legt man bei der Umrechnung auf Proteingehalt willkürlich einen Faktor von 6,25 zugrunde, wie das üblicherweise bei Proteinbestimmungen geschieht, ergab sich für die schutzunwirksame Fraktion $F_{1} G_{75}$ ein relativ geringer Proteinanteil von $65 \%$, während die Fraktion $\mathrm{F}_{2} \mathrm{G}_{75}$ praktisch vollständig aus Protein bestand. Die Fraktion $\mathrm{F}_{2} \mathrm{G}_{50}$ nahm eine Mittelstellung ein. Dieser Betrachtung liegt die zunächst unbewiesene Annahme zu- 
grunde, daß der gemessene Stickstoffgehalt aus Aminosäuren stammt. Tatsächlich zeigte die Hydrolyse der Fraktionen mit $10 \% \mathrm{HCl} / 50 \%$ Ameisensäure für $20 \mathrm{Stdn}$. bei $105^{\circ} \mathrm{C}$ und anschließende Papierchromatographie der Hydrolyseprodukte das Vorliegen von Aminosäuren in einer ähnlichen Verteilung wie sie bei der Hydrolyse von Bovinalbumin angetroffen wird. Eine quantitative Bestimmung der Aminosäure-Zusammensetzung wurde nicht vorgenommen und erscheint erst dann zweckmäßig, wenn die Einzelkomponenten der Fraktionen voneinander getrennt vorliegen.

\begin{tabular}{|c|c|c|}
\hline $\begin{array}{c}\text { getestete Lösung } \\
\text { (Zugaben in der } \\
\text { angegebenen Reihenfolge) }\end{array}$ & $\begin{array}{r}\text { Gefr } \\
\mathrm{i} \\
\mathrm{Tl} \\
\mathrm{be} \\
\mathrm{Be} \\
\mathrm{Pl} \\
{[\mu \mathrm{mo}}\end{array}$ & $\begin{array}{l}\text { der Testlösung } \\
\text { genwart von } \\
\text { oiden } 3 \text { Stdn. } \\
5^{\circ} \text {, anschließ- } \\
\text { Iessung der } \\
\text { orylierung in } \\
\text { Chlor. } \rightarrow \text { tde.] }\end{array}$ \\
\hline \multicolumn{3}{|c|}{$\begin{array}{l}\mathrm{F}_{2} \mathrm{G}_{75}(0,5 \mathrm{mg} / \mathrm{ml} 0,005 \text { м TRIS, } \\
\mathrm{pH} 7,8)\end{array}$} \\
\hline \multicolumn{3}{|c|}{$\mathrm{F}_{2} \mathrm{G}_{75}+$ Trypsininhibitor $(0,5 \mathrm{mg} / \mathrm{ml})$} \\
\hline \multicolumn{3}{|l|}{$\mathrm{F}_{2} \mathrm{G}_{75}+$ Trypsin, nach $10 \mathrm{~min}$} \\
\hline \multirow{2}{*}{\multicolumn{3}{|c|}{$\begin{array}{l}\text { Inkubation + Trypsininhibitor } \\
\mathrm{F}_{2} \mathrm{G}_{75}+\text { Trypsin, nach } 40 \text { min }\end{array}$}} \\
\hline & & \\
\hline Inkubation + Trypsininhibitc & & 43 \\
\hline
\end{tabular}

Tab. 2. Inaktivierung der schutzstoffaktiven Fraktion $\mathrm{F}_{2} \mathrm{G}_{75}$ aus Spinat durch Trypsin. Zugabe von Thylakoiden zum Testsystem nach Hemmung des Trypsins durch Trypsininhibitor.

Trypsinbehandlung verminderte oder beseitigte die Schutzstoffaktivität, wie aus Tab. 2 hervorgeht.

Den Gehalt der Fraktionen an Hexosen, wie er mittels der Anthronmethode gemessen wurde, zeigt Tab. 3. Danach war die unwirksame Fraktion $F_{1} G_{75}$ durch einen hohen Gehalt an Hexosen ausgezeichnet, während der Hexosengehalt der schutzwirksamen Fraktionen gering war und möglicherweise lediglich eine Verunreinigung durch die vorlaufende unwirksame Fraktion darstellt. Der Gehalt an Pentosen wurde mittels der Orcin- und der Bial-Methode ${ }^{12}$ bestimmt (Tab.3). Wieder zeigte die unwirksame Fraktion einen hohen Gehalt an Pentosen. Die schutzwirksamen Fraktionen gaben demgegenüber nur schwache Pentosenreaktion. Um die Spezifität der angewandten Zucker-Bestimmungsmethoden nachzuprüfen, wurden Proben der Fraktionen mit $1 \mathrm{~N}-\mathrm{HCl}$

11 D. L. Morris,Science [New York] 107, 254 [1948].

12 T. H. Ploetz, in: Hoppe-Seyler/Thierfelder, Handbuch der Physiologisch- und Pathologisch-chemischen Analyse III, 1, 714, Springer, Berlin 1955.

\begin{tabular}{lccc}
\hline Material & $\begin{array}{c}\text { Anthron- } \\
\text { Test } \\
\text { (Hexosen) }\end{array}$ & $\begin{array}{c}{[\%] \text { Zucker }} \\
\text { Bial-Test } \\
\text { (Pentosen) }\end{array}$ & $\begin{array}{c}\text { Orcin-Test } \\
\text { (Hexosen } \\
+ \text { Pentos.) }\end{array}$ \\
\hline $\mathrm{F}_{2} \mathrm{G}_{50}$ aus Spinat & 3,1 & 2 & - \\
$\mathrm{F}_{2} \mathrm{G}_{50}$ aus Winter- & 3 & 2,2 & 2,8 \\
$\quad$ roggen & 3 & 2,4 & 1,9 \\
$\mathrm{~F}_{2} \mathrm{G}_{75}$ aus Spinat & 1,3 & 2 & 2,3 \\
$\mathrm{~F}_{2} \mathrm{G}_{57}$ aus Winter- & 3,5 & 22 & 36 \\
roggen & 12,4 & 23 & 44 \\
$\mathrm{~F}_{1} \mathrm{G}_{75}$ aus Spinat & 7,7 & 2,5 \\
$\mathrm{~F}_{1} \mathrm{G}_{75}$ aus Winter- \\
roggen
\end{tabular}

Tab. 3. Der Gehalt der Fraktionen $F_{2} G_{50}, F_{2} G_{75}$ und $F_{1} G_{75}$ an gebundenen Zuckern. Die Anthron-Methode ${ }^{11}$ (Referenz Saccharose) gibt den Gehalt an Hexosen wieder, der BialTest ${ }^{12}$ (Referenz äquimolare Lösung von Ribose und Arabinose) den von Pentosen. Die Orcin-Methode ${ }^{12}$ (Referenz Arabinose) soll Pentosen und Hexosen zusammen erfassen.

60 min bei $100^{\circ} \mathrm{C}$ hydrolysiert und das Hydrolysat nach Entfernen der Salzsäure bei $10^{\circ} \mathrm{C}$ papierchromatographiert. Die Anilinphthalat-Reaktion ${ }^{13}$ ergab bei der Fraktion $\mathrm{F}_{1} \mathrm{G}_{75}$ drei Flecken, wovon einer als Glucose identifiziert wurde, während die beiden anderen die für Pentosen charakteristische Farbreaktion ergaben. Die Hydrolysate der schutzwirksamen Fraktionen zeigten nach chromatographischer Auftrennung nur schwache Reaktion mit Anilinphtalat.

Eine Bilanzbetrachtung (Tab.4) ergibt somit, daß die schutzwirksame Fraktion $\mathrm{F}_{2} \mathrm{G}_{50}$ ganz überwiegend Protein (bzw. Polypeptid) und daneben wenig Zukker enthielt. Ca. $15 \%$ des Gesamtgewichtes sind in der Bilanz nicht erfaßt. $F_{2} G_{75}$ bestand nahezu voll-

\begin{tabular}{|c|c|c|}
\hline \multirow{2}{*}{ Material } & \multicolumn{2}{|c|}{$\begin{array}{c}{[\%] \text { des Gesamtgewichtes }} \\
\text { analytisch erfaßt }\end{array}$} \\
\hline & I & II \\
\hline $\mathrm{F}_{2} \mathrm{G}_{50}$ aus Spinat & 83 & - \\
\hline $\mathrm{F}_{2} \mathrm{G}_{50}$ aus Winterroggen & 85 & 83 \\
\hline $\mathrm{F}_{2} \mathrm{G}_{75}$ aus Spinat & 103 & 101 \\
\hline $\mathrm{F}_{2} \mathrm{G}_{75}$ aus Winterroggen & 103 & 99 \\
\hline $\mathrm{F}_{1} \mathrm{G}_{75}$ aus Spinat & 97 & 99 \\
\hline $\mathrm{F}_{1} \mathrm{G}_{75}$ aus Winterroggen & 95 & 108 \\
\hline ,,F $\mathrm{F}_{2} \mathrm{G}_{50}{ }^{\circ}$ abgetrennt aus & & \\
\hline Pepton Merck & 90 & 89 \\
\hline
\end{tabular}

Tab. 4. Bilanz der bei der Analyse der Fraktionen $\mathrm{F}_{2} \mathrm{G}_{50}$, $\mathrm{F}_{2} \mathrm{G}_{75}$ und $\mathrm{F}_{1} \mathrm{G}_{75}$ erfaßten Substanzen. $\mathrm{I}=\%$ „Protein “ $+\%$ Hexosen (Anthron-Methode) + \% Pentosen (Bial-Test), $\mathrm{II}=\%$ „Protein“ $+\%$ Zucker (Orcin-Methode).

13 S. M. Partridge, Biochem. J. 42, 238 [1948]. 
ständig aus Protein (Polypeptid). Eine Bilanzlücke ergibt sich hier nicht. Die unwirksame Fraktion $\mathrm{F}_{1} \mathrm{G}_{75}$ enthielt den geringsten Anteil an Protein und daneben beträchtliche Mengen an Zuckern. Auch hier ist eine Bilanzlücke nicht vorhanden.

Die Molekulargröße läßt sich zunächst näherungsweise aus dem Verhalten der Fraktionen gegenüber dem auf dem Molekularsiebprinzip trennenden Sephadex abschätzen. Die beiden schutzwirksamen wie auch die inaktive Fraktion wurden nicht von Sephadex G-25 aufgenommen, das globuläre Moleküle bis zu einem Mol.-Gew. von etwa $5000 \mathrm{zu}$ trennen vermag. Eine Trennung erfolgte erst an Sephadex G-50 und Sephadex G-75. Das weist auf höhere Mol.-Gew. hin.

Zur weiteren Abschätzung wurden Sedimentations-Koeffizienten und Diffusionskonstanten in der analytischen Ultrazentrifuge (Modell U 60 L, Phywe, Göttingen) bestimmt. Die erhaltenen Werte wurden durch Extrapolation auf die Konzentration Null und durch Umrechnung auf $20{ }^{\circ} \mathrm{C}$ und Wasser als Lösungsmittel korrigiert. Das partielle spez. Volumen war $0,82 \mathrm{mg} / \mathrm{g}$ (Cahn-Waage, Beckmann Instr.). Die einzelnen Fraktionen wanderten in einem einheitlichen, sich relativ rasch abflachenden Gipfel. Eine Aufspaltung in Subkomponenten wurde nicht beobachtet. Aus den erhaltenen Daten (repräsentative Werte in Tab.5) ergaben sich nach Einsetzen

\begin{tabular}{cccc}
\hline Material & $\begin{array}{c}D_{0}^{20} \\
{\left[10^{-6} \mathrm{~cm}^{2} / \mathrm{sec}\right]}\end{array}$ & $\begin{array}{c}s_{0}^{20} \\
{\left[10^{-13} \mathrm{sec}\right]}\end{array}$ & $\begin{array}{c}\text { Angenähert. } \\
\text { Mol.-Gew. }\end{array}$ \\
\hline $\mathrm{F}_{2} \mathrm{G}_{50}$ & 1,3 & 0,98 & ca. 10000 \\
$\mathrm{~F}_{2} \mathrm{G}_{75}$ & 0,83 & 1,06 & ca. 17000 \\
$\mathrm{~F}_{1} \mathrm{G}_{75}$ & 1,07 & 2,8 & ca. 35000 \\
\hline
\end{tabular}

Tab. 5. Diffusionskonstanten $D$, Sedimentationskoeffizienten $s$ und ungefähre Mol.-Gew. der Fraktionen $\mathrm{F}_{2} \mathrm{G}_{50}$, $\mathrm{F}_{2} \mathrm{G}_{75}$ und $\mathrm{F}_{1} \mathrm{G}_{75}$.

in die Sve dberg- Formel ${ }^{14}$ Mol.-Gew. von etwa 10000 für die aktive Fraktion $F_{2} G_{50}$, von 15000 bis 20000 für $F_{2} G_{75}$ und von ca. 35000 für die unwirksame Fraktion $F_{1} G_{75}$. Die Berechnung setzt eine sphärische Gestalt der Moleküle voraus, ohne daß diese Voraussetzung geprüft werden konnte.

Trotz der einheitlichen Sedimentation der Fraktionen in der Ultrazentrifuge, die eine relativ einheitliche Molekulargröße anzeigte, deuteten die Daten über die Schutzstoffwirkung der aktiven Fraktionen bereits an, daß in ihnen mehr als eine Komponente enthalten ist. Diese Vermutung wird bestä-
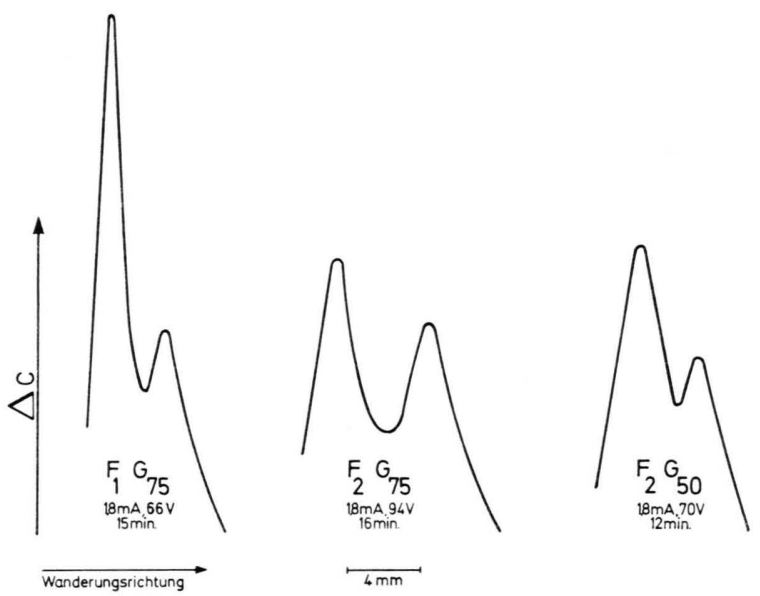

Abb. 11. Wanderung der Schutzstofffraktionen im elektrischen Feld. Mikroelektrophorese nach Antweiler. $\Delta c=$ Konzentrationsänderung gemessen als Änderung des Brechungsindex im Medium.

tigt durch die elektrophoretische Untersuchung. Nach einem interferometrischen Verfahren ausgewertete Elektropherogramme der Fraktionen sind in Abb. 11 dargestellt. Alle drei Fraktionen spalteten in Veronalpuffer ( $\mathrm{pH}$ 8,2) unter den Bedingungen der Flüssigkeits-Elektrophorese in eine rasch und eine langsam wandernde Komponente auf. Die schnelleren Subfraktionen waren in allen Fällen kleiner als die mehr stationären Komponenten.

\section{Diskussion}

Die im Vorstehenden beschriebenen Ergebnisse zeigen, daß die beiden Schutzstofffraktionen als wirksame Komponente hitzestabiles Protein bzw. hochmolekulares Polypeptid enthalten. Die Fraktionen sind nicht homogen und es ist möglich, daß in ihnen mehr als eine aktive Komponente enthalten ist.

Bezüglich der biologischen Bedeutung der Befunde interessieren mehrere Fragen. Reflektieren die im Membrantest erhaltenen Ergebnisse tatsächlich die Wirksamkeit einer Verbindung, die in vivo am Frostschutz einer Zelle ursächlich beteiligt ist? Steht der überraschend hohen Aktivität der Proteinschutzstoffe im Membrantest eine gleich hohe Wirksamkeit in vivo gegenüber? Mehrere Argumente sind hier relevant. Verbindungen, die Erythrozyten oder Spermazellen gegen die Wirkung des Frostes zu schützen vermögen, sind auch im Membrantest aktiv. Der entscheidende Vorteil des Membrantestes gegen-

14 Th. Svedberg u. K. O. Pedersen: The Ultrazentrifuge, Clarendon Press, Oxford 1940. 
über einem in vivo-Test, der intakte Zellen als Indikator einer Schutzstoffwirkung verwendet, besteht darin, daß er ein weitgehend offenes System darstellt. Um intakte Zellen gegen die Effekte des Gefrierens schützen zu können, muß ein Schutzstoff erst Permeabilitäts-Barrieren überwieden. Ineffektivität eines Stoffes im in vivo-System muß nicht notwendigerweise besagen, daß der Stoff schutzunwirksam ist, sondern kann auch bedeuten, daß er lediglich die zu schützenden Stellen nicht oder nicht schnell genug zu erreichen vermag. Demgemäß spricht der Membrantest auch empfindlich gegenüber Verbindungen wie etwa Saccharose an, die als Additiva zu einer Suspension intakter Zellen wenig schutzwirksam sind, es sei denn, daß die Aufnahme in die Zellen sichergestellt ist.

$\mathrm{Da} ß$ die Proteinschutzstoffe auch in vivo hochwirksam sind und eine wesentliche, oft vielleicht eine ausschlaggebende Rolle beim Erwerb der Resistenz durch Pflanzenzellen spielen, kann zunächst nur auf indirekte Weise geschlossen werden. Während eine Reihe von Pflanzen beim Resistenzerwerb lösliche Zucker anhäufen und heute kein Zweifel mehr darüber besteht, daß diese als Schutzstoffe fungieren, geht bei anderen der Resistenzerwerb - ohne daß es zu einer Anhäufung von Zuckern kommen muß mit einem Anstieg leicht löslicher Proteine einher 1, 4,15. Es liegt nahe, hier wenigstens zum Teil eine Akkumulation von Proteinschutzstoffen zu vermuten. Weiter wurde beobachtet, daß isolierte Chloroplasten aus frostharten Spinatblättern auch nach dem Auswaschen schutzstoffaktiver Zucker resistent gegenüber Gefrieren sind ${ }^{5}$. Erst nach Entfernen der Proteinschutzstoffe durch osmotischen Schock werden sie frostempfindlich. Chloroplasten aus nicht resistentem Sommerspinat sind frostempfindlich, ohne daß das Entfernen löslicher Proteine notwendig wäre. Diese Befunde zeigen, daß die Proteinschutzstoffe auch in vivo derart lokalisiert sind, daß sie ihre Schutzfunktion entfalten können. In früheren Untersuchungen konnte gezeigt werden, daß Chloroplasten beim Erfrieren der Pflanzenteile un-

15 D. Siminovitch u. D. R. Briggs, Plant Physiol. 28, 177 [1953]. mittelbar und nicht erst sekundär geschädigt werden (s. l. c. ${ }^{3}$ und unpublizierte Befunde).

Der Nachweis der Existenz von Schutzstoffen gegenüber dem Gefriertod der Zelle, deren Wirksamkeit die bekannter kryoprotektiver Zusätze wie etwa Glycerin oder Dimethylsulfoxyd erheblich übersteigt, mag von potentieller praktischer Bedeutung sein. In der Regel sind hohe Konzentrationen - um 10\% $(\mathrm{w} / \mathrm{v})$ oder mehr - an niedermolekularen Schutzstoffen erforderlich, um intakte Zellen gegen die Wirkung des Gefrierens zu schützen ${ }^{16}$. Im Membrantest mit Thylakoiden werden $2-3 \%$ Saccharose, Glycerin oder Dimethylsulfoxyd für kompletten Schutz benötigt. Demgegenüber genügen $0,1 \%$ der Proteinschutzstoffe für eine weitgehende Konservierung der Membranaktivität. Außerdem vermögen noch erheblich niedrigere Konzentrationen (0,01\%) den für einen kompletten Schutz des Systems erforderlichen Spiegel an Saccharose oder anderen niedermolekularen Schutzstoffen wesentlich zu senken. Allerdings ist hier zu berücksichtigen, daß der Schutzstoff die zu schützenden Membranen erreichen muß, damit ein Schutzeffekt eintreten kann. Die im Membrantest verwendeten Thylakoidvesikel sind auch für einen hochmolekularen Schutzstoff weitgehend zugänglich, da nur die Außenseite der geschlossenen Membransysteme gegen die mit dem Gefrieren einhergehende Entwässerung geschützt werden muß. Ein Schutz intakter Zellen ist schwerer zu erreichen, da ein Eindringen der Schutzstoffe in die Zelle die Voraussetzung für eine hohe Wirksamkeit ist. In orientierenden Versuchen gelang es nicht, die gefrierbedingte Hämolyse von menschlichen Erythrozyten durch Zusatz der Proteinfaktoren zu verhindern.

Wir sind Frau Krüger, Bonn, und Frau von BrevERN, Hamburg, für zahlreiche Isolierungen von Schutzstoffen und für Aktivitätstests zu großem Dank verpflichtet. Herrn Dr. Claes, Bonn, danken wir für die Bestimmung des partiellen spez. Volumens der Schutzstoffe. Die Untersuchungen wurden von der Deutschen Forschungsgemeinschaft gefördert. Ein Teil von ihnen wurde am Institut für Landwirtschaftliche Botanik der Universität Bonn durchgeführt.

16 G. F. Doebbler, A. W. Rowe u. A P. Rinfret, in: Cryobiology, T. H. Meryman ed., S. 407, Academic Press, London 1966. 